Archives

9| 1992

Varia

\title{
Les recherches hispaniques à L'E.H.E.S.S
}

\section{OpenEdition}

Journals

Édition électronique

URL : http://journals.openedition.org/ccrh/2806

DOI : $10.4000 /$ ccrh.2806

ISSN : 1760-7906

Éditeur

Centre de recherches historiques - EHESS

Édition imprimée

Date de publication : 15 avril 1992

ISSN : 0990-9141

Référence électronique

"Les recherches hispaniques à L'E.H.E.S.S », Les Cahiers du Centre de Recherches Historiques [En ligne], 9 | 1992, mis en ligne le 18 mars 2009, consulté le 21 avril 2019. URL : http://journals.openedition.org/ ccrh/2806 ; DOI : 10.4000/ccrh.2806

Ce document a été généré automatiquement le 21 avril 2019

Article L.111-1 du Code de la propriété intellectuelle. 


\section{Les recherches hispaniques à L'E.H.E.S.S}

1 Ces recherches se développent actuellement dans plusieurs directions, couvrant une large période, du Moyen Age à l'époque moderne. Plusieurs équipes y participent, organisées autour d'un thème spécifique. Nous présentons ici ces différents axes de recherche.

\section{La construction de l'État dans l'Espagne médiévale : pouvoir et savoir. l'État et la culture (responsable : Adeline Rucquoi)}

2 Les recherches menées au cours des cinq dernières années sur le thème de la genèse médiévale de l'Espagne moderne ont mis en valeur, entre autres caractéristiques spécifiques à la Péninsule ibérique, l'importance toute particulière accordée par la monarchie à la « sagesse ». Attribut divin comme la justice, la sagesse est effectivement considérée comme un attribut royal dès la fin du XII ${ }^{\mathrm{e}}$ siècle. Cet intérêt porté à la sapientia royale est à l'origine des oeuvres attribuées à certains souverains, tels Alphonse $\mathrm{X}$ ou Sanche IV, qu'ils les aient écrites, dictées ou inspirées. Cette sagesse des rois est également à l'origine des nombreuses oeuvres qui leur sont adressées par des laïcs ou ecclésiastiques cherchant par ce moyen à se concilier leurs bonnes grâces ou obtenir quelque avantage matériel. De ce fait, les souverains jouent un rôle de mécène ou d'instigateur de la culture dans le domaine des arts en général. En fait, dès le XI ${ }^{\mathrm{e}}$ siècle, ils ont mené une véritable "politique» culturelle: abandon du rite et de l'écriture wisigothique pour le rite romain et l'écriture Caroline ; au XII ${ }^{\mathrm{e}}$ siècle, les chanceliers des rois seront des «maîtres », pourvus d'une formation intellectuelle et théologique ; au XIII ${ }^{\mathrm{e}}$ siècle, la monarchie prendra l'initiative de créer des universités et des studia, encourageant les traductions de l'arabe et diffusant le droit romain « importé » de l'école de Bologne; au XIV ${ }^{\mathrm{e}}$ siècle, les letrados, issus des classes moyennes et formés dans les universités, constitueront le noyau de l'Etat moderne en gestation.

3 La sapientia est également un instrument du pouvoir des souverains. Ces derniers exercent un contrôle sur l'enseignement dispensé dans les universités où viennent se former les futurs administrateurs et officiers de la Couronne. Universités qui sont le support d'une politique de propagande qui s'appuie sur les rites, les images, les formules 
utilisées par la chancellerie ou l'élaboration d'une historiographie particulière. Les letrados issus de ces universités leur devront leur ascension sociale et leur fortune aux XIV ${ }^{e}$ et $\mathrm{XV}^{\mathrm{e}}$ siècles. Les membres de l'aristocratie participeront également de cette dynamique et concilieront, en grand nombre, la carrière des armes et celle des lettres. De nombreux nobles - tels Juan Manuel petit-fils du roi Ferdinand III ou le marquis de Santillane écriront, feront écrire des ouvrages, achèteront des livres pour leurs bibliothèques et encourageront les traductions d'auteurs « classiques » latins ou «modernes » français et italiens.

4 Les recherches en cours s'articulent autour des rapports entre le pouvoir et le savoir dans le cadre de la formation de l'État moderne: du savoir "géré » par la Couronne pour asseoir ses revendications et son pouvoir au savoir " utilisé » par certains groupes sociaux pour affirmer leur existence en imposant celle de l'État. La période considérée s'étend $\mathrm{du}$ IX $\mathrm{X}^{\mathrm{e}}$ siècle $\mathrm{au} \mathrm{Xv}^{\mathrm{e}}$ siècle; la Reconquête en effet, élément dominant de la propagande royale dès le $\mathrm{IX}{ }^{\mathrm{e} \text { siècle }}$, contribue à « unifier » l'histoire de la péninsule ibérique dans la longue durée. Ces travaux feront l'objet d'une publication dans le courant de l'année 1993.

5 Participent à cette recherche: Michel Zimmermann (Université de Paris I), Adeline Rucquoi (CNRS-CRH), Martin Aurell (Université de Rouen), Denis Menjot (Université de Strasbourg), Jeanne Allard (EHESS).

\section{L'État espagnol et les Indes occidentales (responsable : Jean-Pierre Berthe).}

Ces travaux portent sur les modalités spécifiques d'exercice de l'institution étatique espagnole aux royaumes d'outre-mer en Amérique. Cette institution étatique est un prolongement de l'Etat castillan, qui doit résoudre divers problèmes: extension territoriale rapide et constitution d'un droit spécifique. Elle ne rencontre aucun obstacle historique si ce n'est le problème de l'encomienda: pas de Cortes, pas de señorios (sauf exception), pas de système de fueros. Mais l'obstacle de l'espace impose un gouvernement à distance. L'exercice du pouvoir est indirect : vice-rois, gouverneurs, audiences, officiers des finances.

7 Dans le domaine ecclésiastique, le patronage royal est absolu : ni le pape ni les patrons particuliers ne peuvent intervenir en matière de nominations ou de dîmes. Contraste avec l'Espagne? Par ailleurs en Espagne, l'Eglise est source de revenus pour le roi ; aux Indes, elle est cause de dépenses.

8 L'éloignement et le sous-encadrement administratif posent le problème de l'application des lois. La corruption et la contrebande sont des phénomènes manifestes : sont-ils plus ou moins importants que dans la Péninsule? Comment magistrats, corregidors, religieux, inquisiteurs exercent-ils leur pouvoir? Quel est le rôle des clientèles locales ainsi que des réseaux de pouvoir qui, à la cour du roi, pèsent sur les décisions politiques et sur les nominations concernant les Indes? Autant de questions qui font l'objet de cette recherche.

9 Participent à cette recherche : Jean-Pierre Berthe (EHESS), Pascal Girard (EHESS), Alain Musset (Université de Paris X), Sylvie Lecoin (Université de Paris VIII), Carmen Val-Jullian (ENS Fontenay), François Vergneault (EHESS). 


\section{Enquête sur la haute administration espagnole au XVIII e siècle}

10 l'une française (Didier Ozanam), l'autre espagnole (Pere Molas, puis Maria Victoria Lopez Cordon). Travail d'archives en Espagne et dépouillements bibliographiques sont menés de front depuis lors. Actuellement, plus de 10.000 hauts fonctionnaires civils, militaires et ecclésiastiques ont été recensés. Si leur nombre désormais varie peu, la quantité d'informations les concernant s'accroît sans cesse. Cette vaste enquête débouche sur les résultats suivants :

11 1- La constitution d'un fichier informatisé à la Maison des pays ibériques à Bordeaux : ce fichier informatisé reste ouvert et intègre au fur et à mesure les nouvelles données recueillies chaque année. Véritable base de données, il permet dès maintenant aussi bien des classements catégoriels que des croisements multiples (réseaux géographiques, familiaux, institutionnels).

2 - La publication de répertoires biographiques par catégories professionnelles et socioprofessionnelles, où des notices alphabétiques résument les informations personnelles, familiales et de carrière des fonctionnaires intéressés. Un premier répertoire établi par Fabrice Abbad et Didier Ozanam est en cours de publication; il concerne les quelque 200 intendants ayant exercé au cours du XVIII ${ }^{e}$ siècle. Un second, déjà bien avancé, établi par Didier Ozanam, recensera les diplomates (environ 400 notices). D'autres sont prévus pour les officiers généraux (les premiers dépouillements ont eu lieu cette année), pour les audiences et chancelleries, les secrétariats d'Etat, les conseils, le haut clergé, etc. Leur mise en oeuvre sera réalisée par des membres des deux équipes travaillant, suivant les cas, séparément ou ensemble. C'est un outil fondamental pour connaître l'arrière-plan familial, humain et financier de ces fonctionnaires, pour évaluer l'ampleur des réseaux de relations et de pouvoir dans lesquels ils s'insèrent et l'influence de ceux-ci sur l'action de l'Etat.

133 - La rédaction d'études institutionnelles, qui font défaut dans plusieurs domaines et sans lesquelles on comprendrait mal le rôle des fonctionnaires recensés. Priorité a été donnée à l'administration financière : après avoir établi une nomenclature des postes de cette administration à la fin du XvIII ${ }^{\mathrm{e}}$ siècle, Jean-Pierre Dedieu a largement entamé la rédaction d'un Dictionnaire institutionnel des finances d'Etat espagnoles. un effort semblable sera fait pour l'administration militaire.

14 Participent actuellement aux travaux de l'équipe française: Didier Ozanam (EHESS), Denise Ozanam (EHESS), Jean-Pierre Dedieu (CNRS), René Quatrefages (CNRS), Claude Larquié (Université de Picardie), Michel Bertrand (Université de Toulouse le Mirail), Martine Seguela (Casa de Velazquez) et un certain nombre d'étudiants. L'équipe espagnole est dirigée par Maria Victoria Lopez Cordon (Université Complutense) et Maria Angeles Perez Samper (Université centrale de Barcelone). 


\section{Les minorités ethniques et religieuses en Espagne et en Amérique latine à l'époque moderne : intégration, exclusion, ségrégation. (responsable : Charles Amiel)}

L'histoire espagnole, tant dans la péninsule qu'en Amérique, offre le cas privilégié d'une coexistence dans la longue durée entre groupes sociaux et cultures de caractères très différents: le monde hispanique est un véritable laboratoire en ce qui concerne l'expérience de l'altérité.

La coexistence séculaire, hostile ou pacifique, sur le sol espagnol de communautés d'origines ethniques et de religions différentes est en effet l'un des traits les plus originaux de l'Espagne médiévale. La communauté musulmane s'y maintient pendant près de huit siècles, l'islam perdant définitivement tout pouvoir avec la chute de Grenade en 1492, année qui fut également marquée par le décret d'expulsion des communautés juives, restée nombreuses et florissantes dans ce pays, et par la découverte de l'Amérique qui apporte la révélation de sociétés qui s'étaient développées pendant des millénaires hors de tout contact avec l'Ancien Monde. L'instauration du système colonial dans les diverses vice-royautés du Nouveau Monde détermine une nouvelle coexistence, séculaire également, entre deux «républiques » : celle des Espagnols d'une part, celle des Indiens de l'autre. En outre, nombre de «nouveaux-chrétiens » (juifs et musulmans nouvellement convertis sous la contrainte) et de leurs descendants ont émigré dans les colonies espagnoles espérant y trouver un refuge.

17 Cette recherche s'attache à l'étude comparée de ces minorités, officiellement catholiques, en Espagne et dans ses possessions d'Amérique $\mathrm{du} \mathrm{XVI}^{\mathrm{e}}$ au $\mathrm{XVIII}^{\mathrm{e}}$ siècle et à l'analyse des processus complexes qui ont visé à leur intégration tout en développant des phénomènes contraires d'exclusion et de ségrégation.

18 Les principales sources utilisées sont les archives inquisitoriales, d'une très grande richesse, ainsi que les archives des ordres religieux, en Espagne, en Amérique ou à Rome, et les archives nobiliaires dans la mesure où elles sont accessibles. Dans une seconde phase, des enquêtes seront menées sur le terrain, au Pérou ou au Mexique, dans des communautés constituées par des descendants de marranes. La recherche s'ordonne autour de deux axes :

- stratégies et pratiques du pouvoir (royal et inquisitorial) visant à l'assimilation des "nouveauxchrétiens".

De nombreux thèmes s'inscrivent dans cet axe ; citons à titre d'exemple : les raisons de l'instauration d'une Inquisition en Castille et de l'expulsion des Juifs, les politiques définies par les textes normatifs et leur application à l'échelle locale, les doctrines et diverses stratégies concernant les minorités, émanant du clergé séculier et des différents ordres religieux, la quantification de la persécution et l'étude des conjonctures, etc.

21 - stratégies, adaptations et réactions des minorités.

Une approche encore « extérieure » porte sur les réseaux familiaux et les solidarités entre « nouveaux-chrétiens » (clientélisme, collusions économiques, relations internationales). Pour une étude interne, une grille des possibles a été établie dont on cherchera à restituer les facteurs et contextes: assimilation recherchée ou feinte, assimilation partielle ou consciente, assimilation inconsciente et syncrétisme, refus de l'assimilation. 

poussée de fièvre du culte immaculiste au XVII ${ }^{e}$ siècle correspond à un moment fort de ferveur religieuse), par ses arrière-pensées politiques, par ce qu'il nous apprend sur la pratique religieuse : culte et propagation des images saintes, quadrillage de l'espace par un foisonnement de sanctuaires, multiplication des miracles, flot de littérature spécifique (mystique, poésies, exempla, récits sur l'origine des sanctuaires et pèlerinages), pratiques 
diverses (rosaires, litanies, "lien d'esclavage »), structures et mises en oeuvre diverses (confréries et congrégations mariales, jésuites, chartreux...), luttes d'influence entre divers vocables (Immaculée contre Notre-Dame du Rosaire, Remedios contre Guadalupe), etc. Cet énoncé non exhaustif permet d'entrevoir la richesse de ce thème.

Il s'agit donc de saisir l'originalité, l'unité et la diversité du monde hispanique à travers un vecteur privilégié. Une attention vigilante est portée à tout ce qui peut souder cette cohésion (les grands moments comme les juras à tel vocable de la Vierge), comme à ce qui peut l'affaiblir (révoltes millénaristes et mouvements de résistance dans lesquels le culte marial aura son importance, comme au Chapias en 1712.

Participent à cette recherche : Jean-Pierre Berthe (EHESS), Thomas Calvo (EHESS), Sophie Coussemaker (Casa de Velazquez), Jacques Gelis (Université de Paris VIII), Isabelle Poutrin (Université de Paris XII), Bernard Vincent (EHESS).

\section{École française de Rome} subvention du Ministère de la Recherche, l'équipe compte exploiter de manière systématique les visites ad limina Apostolorum. Celles-ci ont été instituées en 1585 par le pape Sixte Quint. Les prélats étaient tenus de se rendre à Rome tous les trois, cinq ou dix ans selon leur éloignement pour rendre compte de l'état de leur diocèse. Dans les faits, les évêques se déplacèrent rarement mais ils envoyèrent souvent un rapport. L'ensemble de ces rapports se trouve aux archives du Vatican où ils sont consultables pour les archives antérieures à 1890, ce qui constitue un ensemble important couvrant trois siècles.

Ces documents sont de qualité inégale mais certains foisonnent d'informations extrêmement riches concernant l'histoire du diocèse, son étendue, ses privilèges, l'activité pastorale de l'évêque, la situation du clergé séculier et régulier, l'état matériel $\mathrm{du}$ diocèse avec inventaire des églises, des monastères, des hôpitaux, des reliques, des statues de saints, les miracles, etc. Une matière remarquable susceptible de faire l'objet d'une grande enquête.

31 Il s'agit d'un projet de recherche à caractère international rassemblant des chercheurs français, espagnols, italiens, dont les équipes sont en cours de constitution. Le dépouillement des archives concernant ces visites permettrait d'aboutir dans un premier temps à un livre-guide, puis à un atlas d'histoire religieuse et à de nombreuses études comparatives en histoire et anthropologie religieuse.

\section{Nouvelle équipe}

Enfin une équipe de chercheurs en histoire contemporaine est en cours de constitution. Son responsable est Carlos Serrano. Elle se propose de travailler sur Modernité et modernisations dans l'Espagne des $\mathrm{XIX}^{\mathrm{e}}$ et $\mathrm{XX}^{\mathrm{e}}$ siècles. 